\title{
Optical Properties of Mn-doped GaN
}

\author{
O. Gelhausen ${ }^{1}$, E. Malguth ${ }^{1,3}$, M. R. Phillips ${ }^{1}$, E. M. Goldys ${ }^{2}$, M. Strassburg ${ }^{3,4}$, A. Hoffmann ${ }^{3}$, \\ T. Graf ${ }^{5}$, M. Gjukic ${ }^{5}$ and M. Stutzmann ${ }^{5}$ \\ ${ }^{1}$ Microstructural Analysis Unit, University of Technology, Sydney, Broadway, NSW 2007 \\ Australia \\ ${ }^{2}$ Division of Information and Communication Sciences, Macquarie University, North Ryde, \\ NSW 2109, Australia \\ ${ }^{3}$ Institute for Solid-State-Physics, Technical University Berlin, 10623 Berlin, Germany \\ ${ }^{4}$ Department of Physics and Astronomy, Georgia State University, Atlanta, GA-30303, USA \\ ${ }^{5}$ Walter Schottky Institute, Technical University Munich, 85748 Garching, Germany
}

\section{ABSTRACT}

Molecular beam epitaxy-grown GaN with different Mn concentrations $\left(5-23 \times 10^{19} \mathrm{~cm}^{-3}\right)$ and codoped with $\mathrm{Si}$ were investigated by cathodoluminescence (CL) spectroscopy and optical transmission measurements. In the GaN:Mn, an intense absorption peak at $1.414+/-0.002 \mathrm{eV}$ was observed. This peak was attributed to an internal ${ }^{5} \mathrm{~T}_{2} \rightarrow{ }^{5} \mathrm{E}$ transition of the deep neutral $\mathrm{Mn}^{3+}$ state since its intensity scaled with the $\mathrm{Mn}^{3+}$ concentration. The CL measurements showed that Mn-doping concentrations around $10^{20} \mathrm{~cm}^{-3}$ had three effects on the emission spectrum: (i) the donor bound exciton at $3.460 \mathrm{eV}$ was reduced by more than one order of magnitude, (ii) the donor-acceptor-pair band at $3.27 \mathrm{eV}$ was completely quenched and (iii) the yellow luminescence centered at $2.2 \mathrm{eV}$ was the strongly decreased. The latter two effects were attributed to a reduced concentration of $\mathrm{V}_{\mathrm{Ga}}$. In the infrared spectral range, three broad, Mn-doping related CL emission bands centered at $1.01 \pm 0.02 \mathrm{eV}, 1.09 \pm 0.02 \mathrm{eV}$ and $1.25 \pm 0.03 \mathrm{eV}$ were observed. These bands might be related to deep donor complexes, which are generated as a result of the heavy Mn-doping, rather than internal transitions at the $\mathrm{Mn}$ atom.

\section{INTRODUCTION}

Transition metals in $\mathrm{GaN}$ have been studied in detail for more than 10 years [1], but regained attraction recently due to potential application for growth of high-resistivity GaN substrate material and for dilute magnetic semiconductors (DMS) showing ferromagnetic behaviour above room temperature [2]. GaN doped with $\mathrm{Mn}$ is a possible candidate for spintronics applications since a Curie temperature above room temperature has been reported $[3,4]$. However, a viable spintronics device needs to support carrier-mediated ferromagnetism so that spin-polarised charge carriers may be injected into a nonmagnetic semiconductor [5]. Recent reports indicate that the $\mathrm{Mn}^{2+}$ acceptor level is located deep in the GaN band gap and not effective-mass-like as in GaAs:Mn [6], suggesting that carrier-mediated ferromagnetism in GaN:Mn is rather unlikely [7].

Optical studies represent one approach to identify the nature of deep levels, which are generated by the introduction of $\mathrm{Mn}$ in GaN. In this study, low temperature optical absorption measurements and cathodoluminescence (CL) spectroscopy were carried out to investigate the optical characteristics of GaN:Mn and GaN:Mn:Si layers. The aim of this work was to improve the understanding of how the manganese is incorporated into the GaN layers and how this process affects the defect structure of these films. 


\section{EXPERIMENTAL DETAILS}

The $1 \mu \mathrm{m}$ thick samples under investigation were grown by plasma-induced molecular beam epitaxy on c-plane $\mathrm{Al}_{2} \mathrm{O}_{3}$ substrate. The samples were either nominally undoped, $\mathrm{Mn}$ doped with Mn concentrations of $0.5-2.3 \times 10^{20} \mathrm{~cm}^{-3}$, as determined by eleastic recoil detection, or intentionally codoped with $\mathrm{Mn}$ and $\mathrm{Si}\left([\mathrm{Mn}] \sim[\mathrm{Si}] \sim 3 \times 10^{19} \mathrm{~cm}^{-3}\right)$. The absorption measurements were performed at $2 \mathrm{~K}$ with a $250 \mathrm{~W}$ tungsten-halogen lamp and spectrally dispersed by a $0.75 \mathrm{~m}$ double-grated monochromator with a resolution of $0.02 \mathrm{~nm}$. A cooled Ge detector was used to detect the sample transmittance. The CL measurements were performed using an Oxford Instruments MonoCL2 system installed on a JEOL35C SEM. The UV-VIS CL spectra were collected using a 1200 lines/mm grating blazed at $500 \mathrm{~nm}$ and a Hamamatsu R94302 Peltier cooled photomultiplier tube (PMT). The IR spectra were measured using a 600 lines/mm grating blazed at $1600 \mathrm{~nm}$ and a Hamamatsu R5509-72 PMT. All CL spectra were corrected for system response. The electron beam current, $\mathrm{I}_{\mathrm{b}}$, was measured using a Faraday cup.

\section{DISCUSSION}

In order to elucidate the nature of the Mn dopant in $\mathrm{GaN}$, optical absorption measurements of nominally undoped GaN, GaN:Mn and GaN:Mn:Si were carried out at $2 \mathrm{~K}$ (Fig. 1). In the spectra of the purely Mn-doped GaN, an intense absorption peak at $1.414 \pm 0.002$ $\mathrm{eV}$ was observed. This line was not setected in either the nominally undoped or the GaN:Si:Mn samples. The relative intensity of this absorption peak was found to scale with the Mn concentration indicating a transition related to a Mn center. The absorption peak was recently attributed either to the internal ${ }^{5} \mathrm{~T}_{2} \rightarrow{ }^{5} \mathrm{E}$ transition of the deep neutral $\mathrm{Mn}^{3+}$ state [7] or to the optical transition from the valence band to the $\mathrm{Mn}^{2+}$ acceptor $[8,9]$. If the latter interpretation was correct, a strongly pronounced absorption peak would be expected in $\mathrm{GaN}: \mathrm{Mn}: \mathrm{Si}$, where a much higher (more than one order of magnitude) concentration of the $\mathrm{Mn}^{2+}$ acceptors was measured by ESR [7]. The absence of this line in GaN:Mn:Si contradicts the latter interpretation and makes the former assignment more likely.

Based on a comparison with the position of the $\mathrm{Mn}^{2+}$ acceptor level (with respect to the vacuum energy) in $\mathrm{GaAs}, \mathrm{GaP}$ and $\mathrm{InP}$, the $\mathrm{Mn}^{2+}$ level in $\mathrm{GaN}$ is expected at $2.0 \pm 0.1 \mathrm{eV}$ above the valence band [10]. Therefore, the ansorption peak is more likely related to the neutral $\mathrm{Mn}^{3+}$ acceptor state. Due to the splitting of the five $\mathrm{d}$ orbitals of the $\mathrm{Mn}^{3+}$ into three $\mathrm{t}_{2}$ and two e orbitals in the tetrahedral crystal field of the surrounding $\mathrm{N}$ ligands, one (spin-allowed) transition $\mathrm{T}_{2} \rightarrow \mathrm{E}$ is expected in optical absorption spectra. However, in $\mathrm{GaN}$ the $\mathrm{t}_{2}$ orbitals of $\mathrm{Mn}^{3+}$ hybridise with the $\mathrm{p}$ orbitals of the valence band, shifting the occupied bonding states $\mathrm{t}^{\mathrm{b}}$ into the valence band and the antibonding $\mathrm{t}^{\mathrm{a}}$ states above the two nonbonding e states [11]. Calculation based on the tight-binding linear-muffin-tin-orbital method indicated an energy difference, $\Delta \mathrm{E}$, between the gap states of $\Delta \mathrm{E}=\mathrm{E}_{\mathrm{t} 2 \mathrm{~g}}-\mathrm{E}_{\mathrm{eg}} \sim 1.35 \mathrm{eV}$ for zincblende GaN:Mn [12], while calculations within the local spin density approximation gave a respective energy difference of approximately $1.2-1.3 \mathrm{eV}$ for the wurtzite structure of GaN:Mn [11], which roughly agree with the experimentally observed absorption peak at $1.414 \mathrm{eV}$. Thus, we attribute this absorption line to an internal ${ }^{5} \mathrm{~T}_{2} \rightarrow{ }^{5} \mathrm{E}$ transition of the deep neutral $\mathrm{Mn}^{3+}$ state, in agreement with previous reports $[7,13]$. The series of satellite peaks on the higher energy side of the ZPL with an energetic distance of $20 \pm 1 \mathrm{meV}$ is attributed to pseudolocal vibrational modes of the Mn level [8]. 
Figure 1. Optical absorption spectra at $2 \mathrm{~K}$ for MBE-grown undoped, Mn-doped and Mn:Si codoped $\mathrm{GaN}$. The Mn-concentration varied from 5.4 to $23 \times 10^{19} \mathrm{~cm}^{-3}$. The ZPL is at $1.414 \pm 0.002 \mathrm{eV}$. The spectra were corrected from $\lambda_{\text {air }}$ to $\lambda_{\text {vacuum }}$ and calibrated using the 8853.87 $\AA$ and $9148.68 \AA$ lines of a $\mathrm{Ne}$ spectral lamp.

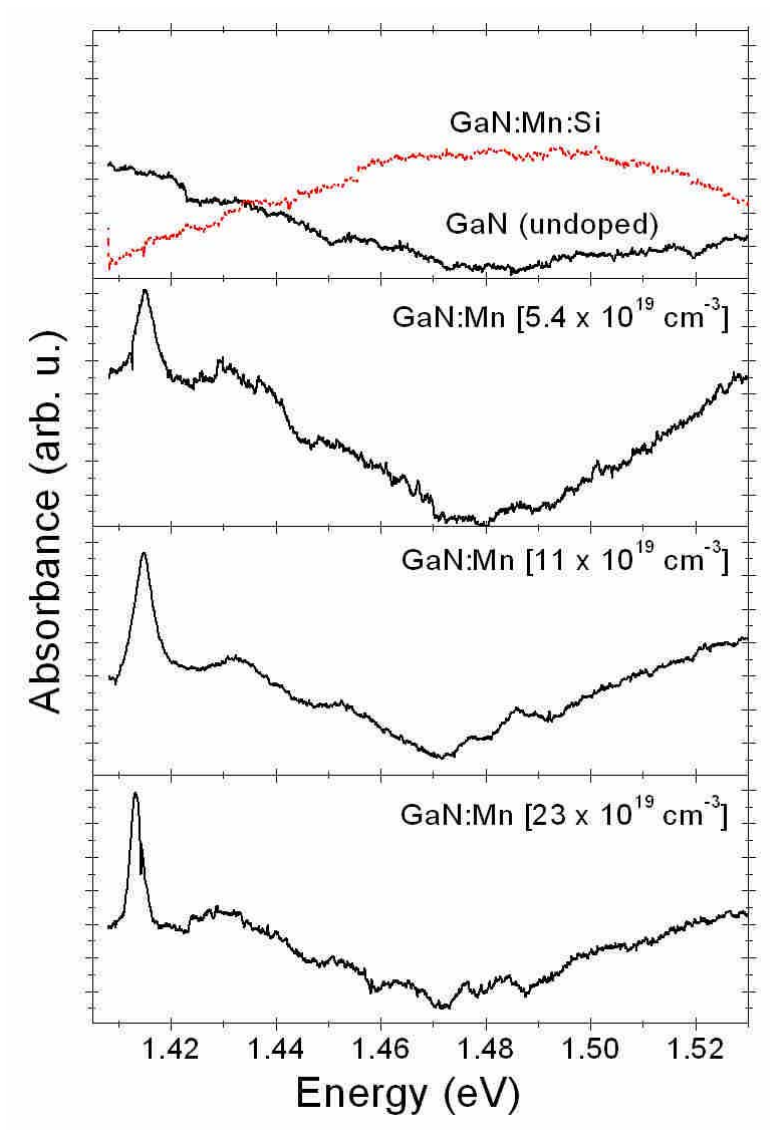

The luminescence properties of nominally undoped GaN, GaN:Mn and GaN:Mn:Si were investigated by CL spectroscopy. It should be noted that the purely Mn-doped samples were charging when irradiated with the electron beam during CL [14], indicating a semi-insulating character due to doping with deep Mn acceptors. A comparison of the absolute intensities of the doped and undoped samples is presented in Fig 2. Mn-doping concentrations around $10^{20} \mathrm{~cm}^{-3}$ reduce the donor bound exciton $\left(\mathrm{D}^{0} \mathrm{X}\right)$ emission intensity by almost one order of magnitude. Moreover, the shallow donor-acceptor-pair (DAP) emission centred at $3.27 \mathrm{eV}$ with its characteristic LO phonon replica [15] is completely quenched and yellow luminescence (YL) around 2.1-2.2 eV [16] is strongly reduced in the Mn-doped samples. The Mn doping promotes nonradiative recombination and effectively quenches the CL in visible part of the spectrum. In addition, the introduction of $\mathrm{Mn}_{\mathrm{Ga}}$ acceptors reduces the concentration of $\mathrm{V}_{\mathrm{Ga}}$ in two ways. First, Mn occupies the Ga lattice site and second, the downshifting of the position of Fermi-level to the midgap region results in a higher formation energy of residual $\mathrm{V}_{\mathrm{Ga}}$ acceptors [17]. The $\mathrm{V}_{\mathrm{Ga}}$ are suggested to be responsible the YL [17,18] and to form acceptor complexes involved in the 3.27 eV DAP in nominally undoped GaN [19].

However, Fig. 2 reveals two additional features: (i) the GaN:Mn:Si sample exhibits a broad multipeak emission band between 3.2 and $3.5 \mathrm{eV}$ and (ii) the onset of an infrared emission band in both the doped and the codoped GaN. The former feature is attributed to an accumulation of defect centers in the GaN:Mn:Si and currently under investigation. 
Figure 2. CL spectra $\left[\mathrm{E}_{\mathrm{b}}=8 \mathrm{keV}, 45 \mu \mathrm{m}\right.$ $\mathrm{x} 36 \mu \mathrm{m}]$ at $80 \mathrm{~K}$ for undoped $\mathrm{GaN}$ (solid line), GaN:Mn $\left[5.4 \times 10^{19} \mathrm{~cm}^{-3}\right]$ (dashed line), GaN:Mn [23 x $10^{19} \mathrm{~cm}^{-3}$ ] (dotted line) and GaN:Mn codoped with $\mathrm{Si}$ (dash-dotted line). The spectra were collected at $20 \mathrm{nA}$ (a), and $5 \mathrm{nA}$ (b,c). Spectrum (a) was devided by a factor of 4. While spectrum c) was collected without any filter, a $400 \mathrm{~nm}$ and a $650 \mathrm{~nm}$ filter were used for acquisition of spectra b) and a), respectively.

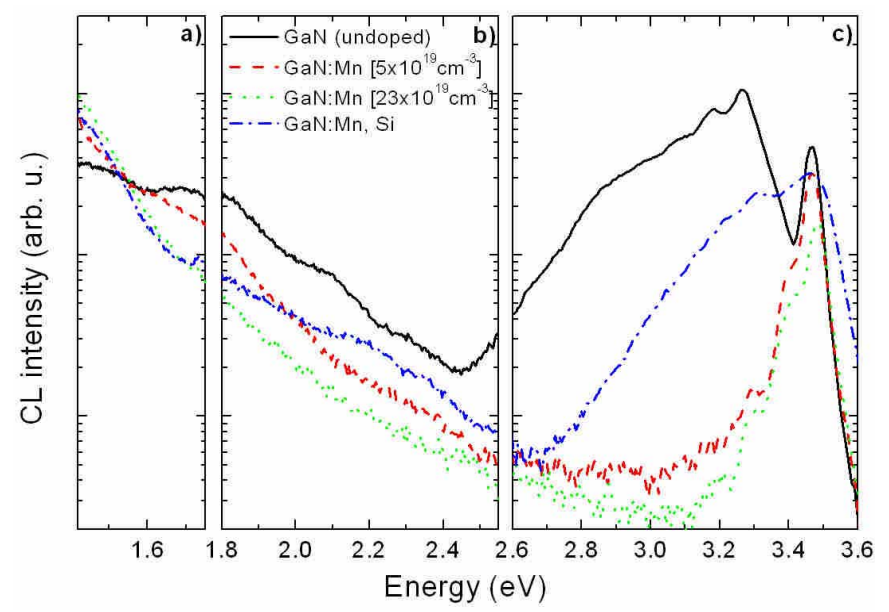

In the infrared spectral range of $0.8-1.4 \mathrm{eV}$ three broad, Mn-doping related CL emission bands centered at $1.01 \pm 0.02 \mathrm{eV}, 1.09 \pm 0.02 \mathrm{eV}$ and $1.25 \pm 0.03 \mathrm{eV}$ were observed (Figs. 3 and 4), which were found to be highly beam sensitive. Continuous electron exposure resulted in an increased intensity, facilitating the determination of the energetic peak position by subtracting the spectra from each other (Fig. 4). The luminescence band at $1.25 \pm 0.03 \mathrm{eV}$ was recently attributed to the spin-forbidden ${ }^{4} \mathrm{~T}_{1}(\mathrm{G})-{ }^{6} \mathrm{~A}_{1}(\mathrm{~S})$ transition at the $\mathrm{Mn}^{2+} \mathrm{d}^{5}$ ion [20]. However, the GaN:Mn with the highest doping level displayed a much stronger IR emission intensity than $\mathrm{GaN}: \mathrm{Mn}, \mathrm{Si}$, which has a one order of magnitude higher concentration of $\mathrm{Mn}^{2+}$. Moreover, neither a ZPL nor the fine structure, which is typical for internal transitions, was observed and the broad shape of this band is rather an indication for deep defects with strong phonon coupling.

In the light of these results, we propose the following model to account for the IR emission bands. Heavy Mn-doping (>3-5 x $10^{19} \mathrm{~cm}^{-3}$ ) leads to a downshift of the Fermi-level and promotes the formation of compensating donor-like defect complexes [21] (e.g. Mn-H [22] and $\mathrm{Mn}-\mathrm{V}_{\mathrm{N}}$ ), i.e. doping-driven self-compensation mechanisms similar to conventional $\mathrm{Mg}$ doping in $\mathrm{GaN}$ [23]. Although theoretical calculations for Mn-related complexes are not available yet, our results indicate that these complexes form deep donor levels, probably with a strong phonon coupling. This is supported by the strong electron beam sensitivity of the IR emission bands, which could be understood in terms of defect complex dissociation [24,25]. Thus, in GaN:Mn codoped with $\mathrm{Mg}$ an even more pronounced IR emission intensity is expected since $\mathrm{Mg}$ codoping will further downshift the Fermi-level and promote the generation of compensating donors. Recently, this was indeed observed in PL experiments on GaN:Mn:Mg and tentatively attributed to $\mathrm{Mn}^{3+}$-donor and $\mathrm{Mn}^{2+}$-donor complexes [20]. Finally, it should be noted that the IR peaks in Fig. 4 cannot stem from the sapphire underneath, since (i) the $2^{\text {nd }}$ order of the $\mathrm{Al}_{2} \mathrm{O}_{2}: \mathrm{Cr}^{3+}$ peak at $0.893 \mathrm{eV}$ is always the strongest sapphire emission in our samples and cannot be observed in the doped samples and (ii) nearly all CL signal is generated within the GaN layer for an electron beam energy of $12 \mathrm{kV}$ [26]. 


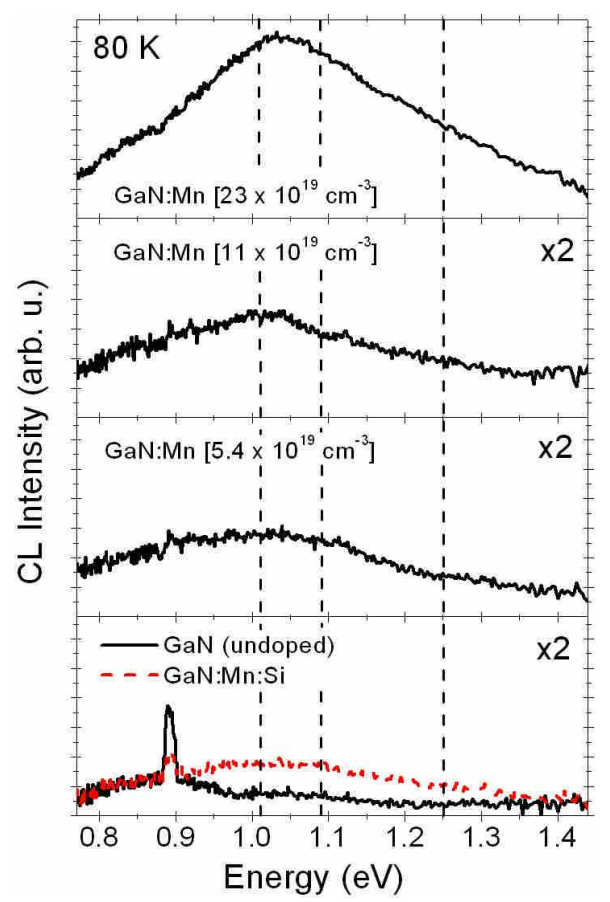

Figure 3. $80 \mathrm{~K} \mathrm{CL}$ spectra $\left[\mathrm{E}_{b}=12 \mathrm{keV}\right.$, $\left.\mathrm{I}_{\mathrm{b}}=150 \mathrm{nA}, 67 \mu \mathrm{m} \times 53 \mu \mathrm{m}\right]$ in the nearinfrared region of undoped, Mn-doped and $\mathrm{Mn}: \mathrm{Si}$ codoped GaN. Compared with the intensity of the top spectrum all other spectra are multiplied by a factor of 2 . The Mn-doping related CL emission bands centered at $1.01 \pm 0.02 \mathrm{eV}, 1.09 \pm 0.02 \mathrm{eV}$ and $1.25 \pm 0.03$ $\mathrm{eV}$ are marked by dashed lines.

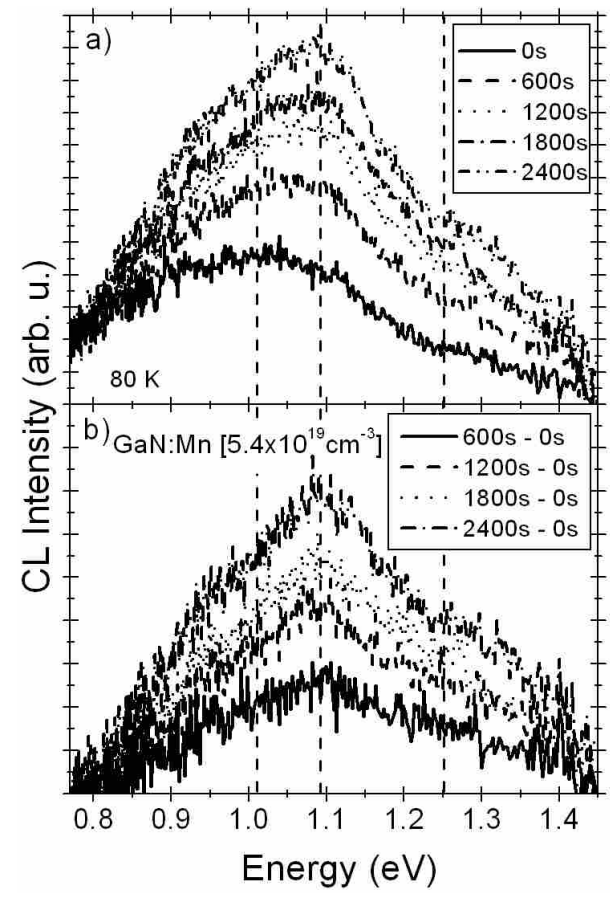

Figure 4. a) The change of the CL spectra during electron beam irradiation with a relatively high dose is illustrated exemplarily for the GaN doped with Mn-concentration of $5.4 \times 10^{19} \mathrm{~cm}^{-3}$. b) Successive changes of the CL spectra, expressed as the difference between the first spectrum and each of the following spectra.

\section{CONCLUSIONS}

In purely Mn-doped $\mathrm{GaN}$, an absorption peak at $1.414+/-0.002 \mathrm{eV}$ was observed at $2 \mathrm{~K}$, which was attributed to an internal ${ }^{5} \mathrm{~T}_{2} \rightarrow{ }^{5} \mathrm{E}$ transition of the deep neutral $\mathrm{Mn}^{3+}$ state and was found to scale in intensity with the $\mathrm{Mn}^{3+}$ concentration. The $\mathrm{CL}$ measurements revealed that Mn-doping concentrations around $10^{20} \mathrm{~cm}^{-3}$ reduced the donor bound exciton emission intensity by almost one order of magnitude. Moreover, the shallow donor-acceptor-pair band at $3.27 \mathrm{eV}$ was completely quenched, whereas the yellow luminescence was strongly decreased. These effects were attributed to the reduced concentration in $\mathrm{V}_{\mathrm{Ga}}$. In the infrared spectral range, three broad, Mn-doping related CL emission bands centered at $1.01 \pm 0.02 \mathrm{eV}, 1.09 \pm 0.02 \mathrm{eV}$ and $1.25 \pm$ $0.03 \mathrm{eV}$ were observed. Their origin is tentatively attributed to deep donor complexes, which are generated as a result of the heavy Mn-doping. 


\section{REFERENCES}

1. B.K. Meyer, A. Hoffmann and P. Thurian, Defect spectroscopy in the nitrides, in B. Gil (ed.) Physics and Applications of Group III Nitride Semiconductor Compounds, Oxford University Press (1997), 242-306

2. T. Dietl, H. Ohno, F. Matsukura, J. Cibert and D. Ferrand, Science 287, 1019 (2000)

3. M. L. Reed, N. A. El-Masry, H. H. Stadelmaier, M. K. Ritums, M. J. Reed, C. A. Parker, J. C. Roberts and S. M. Bedair, Appl. Phys. Lett. 79, 3473 (2001)

4. T. Sasaki, S. Sonoda, Y. Yamamoto, K. Suga, S. Shimizu, K. Kindo and H. Hori, J. Appl. Phys. 91, 7911 (2002)

5. L. Kronik, M. Jain and J. R. Chelikowsky, Phys. Rev. B 66, 41203 (2002)

6. J. Schneider, U. Kaufmann, W. Wilkening, M. Baeumler, F. Kohl, Phys. Rev. Lett. 59, 240 (1987)

7. T. Graf, M. Gjukic, M. S. Brandt, M. Stutzmann and O. Ambacher, Appl. Phys. Lett. 81, 5159 (2002)

8. R. Y. Korotkov, J. M. Gregie and B. W. Wessels, Appl. Phys. Lett. 80, 1731 (2002)

9. S. S. A. Seo, M. W. Kim, Y. S. Lee, T. W. Noh, Y. D. Park, G. T. Thaler, M. E. Overberg, C. R. Abernathy and S. J. Pearton, Appl. Phys. Lett. 82, 4749 (2003)

10. J. Baur, U. Kaufmann, M. Kunzer, J. Schneider, H. Amano, I. Akasaki, T. Detchprohm and K. Hiramatsu, Materials Science Forum 196-201, 55 (1995)

11. H. Katayama-Yoshida and K. Sato, Physica B 327, 337 (2003)

12. E. Kulatov, H. Nakayama, H. Mariette, H. Ohta, Yu. A. Uspenskii, Phys. Rev. B 66, 45203 (2002)

13. T. Graf, M. Gjukic, M. Hermann, M. S. Brandt, M. Stutzmann, L. Görgens, J. B. Philipp and O. Ambacher, J. Appl. Phys. 93, 9697 (2003)

14. To avoid electron beam induced charging, the samples were coated with a thin carbon layer.

15. R. Dingle and M. Ilegems, Solid State Commun. 9, 175 (1971)

16. T. Ogino and M. Aoki, Jpn. J. Appl. Phys. 19, 2395 (1980)

17. J. Neugebauer and C. G. Van de Walle, Appl. Phys. Lett. 69, 503 (1996)

18. K. Saarinen, T. Laine, S. Kuisma, J. Nissila, P. Hautojarvi, L. Dobrzynski, J. M. Baranowski, K. Pakula, R. Stepniewski, M. Wojdak, A. Wysmolek, T. Suski, M. Leszczynski, I. Grzegory and S. Porowski, Phys. Rev. Lett. 79, 3030 (1997)

19. B. Monemar, Journal of Crystal Growth 189/190, 1 (1998)

20. R. Y. Korotkov, J. M. Gregie, B. Han and B. W. Wessels, Physica B 308-310, 18 (2001)

21. C. G. Van de Walle, Phys. Rev. B 56, R10020 (1997)

22. S. Podsiadlo, T. Szyszkoa, G. Warsoa, A. Turos, R. Ratajczak, A. Kowalczyk, W. Gebicki, I. Strzalkowski, D. Grambole, F. Hermann, Vacuum 70, 207 (2003)

23. U. Kaufmann, P. Schlotter, H. Obloh, K. Köhler, and M. Maier, Phys. Rev. B 62, 10867 (2000)

24. H. Amano, M. Kito, K. Hiramatsu, and I. Akasaki, Jpn. J. Appl. Phys. 28, L2112 (1989)

25. O. Gelhausen, M. R. Phillips, H. N. Klein, and E. M. Goldys, Appl. Phys. Lett. 81, 3747 (2002)

26. P. Hovington, D. Drouin, and R. Gauvin, Scanning 19, 1 (1997) 